\title{
Gutachterliste \\ Psychologische Rundschau 2020
}

\author{
An der Gestaltung des Jahrgangs 2020 der Psychologischen Rundschau haben die \\ folgenden Personen durch Gutachten, Stellungnahmen und Revisionsvorschläge \\ entsprechend beigetragen:
}

Andrea Abele-Brehm, Nürnberg-Erlangen

Aljoscha Neubauer, Graz

Urs Baumann, Salzburg

Harald Baumeister, Ulm

Rainer Bromme, Münster

Karin De Santis, Bremen

Stephan Dutke, Münster

Jochen Fahrenberg, Freiburg

Jürgen Glaser, Innsbruck

Andreas Glöckner, Köln

Klaus Fiedler, Heidelberg

Oliver Kliegl, Regensburg

Cornelius König, Saarbrücken

Stephan Kröner, Erlangen

Veit Kubik, Halle

Tanja Lischetzke, Landau

Philipp Mayring, Klagenfurt
Matthias Nückles, Freiburg

Alexander Renkl, Freiburg

Ulf-Dietrich Reips, Konstanz

Rainer Reisenzein, Greifswald

Julian Roelle, Bochum

Christel Salewski, Hagen

Silvia Schneider, Bochum

Peter Schulz, Lugano

Wolfgang Schönpflug, Berlin

Mark Stemmler, Erlangen

Holger Steinmetz, Trier

Ulrich Wagner, Marburg

Oliver Wilhelm, Ulm

Martin Wieser, Berlin

Matthias Ziegler, Berlin

https://doi.org/10.1026/0033-3042/a000516 